incidence of complications, and the index assessing the efficacy of premature ventricular beats, left ventricular systolic function and immune injury of viral myocarditis patients; (2) the assessment index reflecting the myocardial fibrosis, such as carboxyterminal propeptide of type I procollagen (PICP), carboxyterminal propeptide of type III procollagen (P III NP), hyaluronic acid (HA), laminin protein (LN), and Left Ventricular Remodelling Index; (3) the assessment index reflecting the quality of life, such as generic scale and disease-specific scale; (4) the assessment index reflecting the characteristic of Traditional Chinese medicine, for example, syndrome integral scale, which is not sufficient, unified and acknowledged by now. Assessment index of TCM curative effect should reflect the characteristics of TCM which consist of holism concept and syndrome differentiation and treatment. Currently, some scholars try to establish a domain system named as clinical outcome scale reporting by Chinese doctors', laying the foundation for the establishment of assessment method and index system of curative effect which consistent with TCM characteristics.

Conclusions In terms of clinical indicators selection, the assessment index of curative effect of myocardial fibrosis should be as sensitive, specific, acknowledged as possible, and attention should be paid to the complimentarily of indicators; in respect of the establishment of clinical indicator system, attention should be paid to the constitutive and hierarchical properties of indicators, to establish a comprehensive evaluation method with decided significance of each indicator and their relationship.

\section{GW23-e0543 STUDY ON ASSESSMENT INDEX OF CURATIVE EFFECT OF MYOCARDIAL FIBROSIS BASED ON CLINICAL LITERATURE}

doi:10.1136/heartjnl-2012-302920a.285

${ }^{1}$ Lv Shi-chao, ${ }^{1}$ Wu Mei-fang, ${ }^{2} Z$ hu Ya-ping, ${ }^{2}$ Zhang Jun-ping. ${ }^{1}$ Tianjin University of Chinese Medicine; ${ }^{2}$ The First Hospital Affiliated to Tianjin University of Chinese Medicine

Objectives To collate the assessment index of efficacy of myocardial fibrosis and its related problems, and explore the assessment system of curative effect of myocardial fibrosis by means of literature review.

Methods With the application of basic research method of literature review, the clinical literature of myocardial fibrosis is taken as the object of study, and the data of assessment index of curative effect is extracted and normalised, to establish a systematic analysis library of clinical treatment literature.

Results Abnormal heart tissue reconstruction characterised by myocardial fibrosis is the core of pathological changes of a variety of chronic cardiovascular disease (such as hypertension, myocardial infarction, viral myocarditis, etc.), and the primary cause of their persistent, recurrent and permanent uncovered state. At present, the assessment index of curative effect of myocardial fibrosis can be divided into the following categories: (1) the assessment index reflecting curative effect of disease which cause the myocardial fibrosis, such as the index evaluating the step-down rate of hypertension, blood pressure load and blood pressure trough/peak ratio, the circadian rhythm of blood pressure, the index evaluating the mortality of myocardial infarction, cardiovascular events, the 